\title{
Residual stress in geometric features subjected to laser shock
}

\author{
peening \\ M. Achintha ${ }^{1 *}$, D. Nowell ${ }^{2}$ \\ ${ }^{1}$ Engineering and the Environment, University of Southampton, UK \\ ${ }^{2}$ Department of Engineering Science, University of Oxford, UK
}

* Corresponding author:

Engineering and the Environment, University of Southampton, Southampton, SO17 1BJ, UK. Email: Mithila.Achintha@ soton.ac.uk; Telephone: 02380592924

\begin{abstract}
This paper reports selected findings from a collaborative research study into the fundamental understanding of laser shock peening (LSP), when applied to key airframe and aero-engine alloys. The analyses developed include explicit simulations of the peening process together with a simpler eigenstrain approach, which may be used to provide an approximation to the residual stress field in a number of geometries. These are chosen to represent parts of structural components under conditions relevant to service applications. The paper shows that the eigenstrain approach can provide good approximations to the stress field in most circumstances and may provide a computationally efficient tool for exploring different peening strategies. Both explicit and eigenstrain results demonstrate that the interaction between the LSP process and geometric features is important for understanding the subsequent performance of components. Particularly relevant for engineering applications is that not all instances of LSP application may provide an improvement in structural integrity.
\end{abstract}

\section{Keywords}

Eigenstrain, Fatigue, Laser shock peening, Residual stress 


\section{Introduction}

Laser shock peening (LSP) uses Nd:glass laser systems to create a residual surface stress distribution in metallic components ${ }^{1}$. The method involves firing laser pulses at the surface of a component to introduce a compressive residual stress. The present authors have previously attempted to develop a practical predictive tool for the induced stresses, based on the use of eigenstrains (i.e. misfit strains) in a finite element (FE) model $^{1-3}$. The results illustrate that the underlying misfit strain field representing a LSP application is predominantly dependent on the characteristics of the laser pulse and the amount of coverage, rather than the exact geometry of the component. Consequently, the residual stresses produced by LSP can be estimated by using static FE models and previously-derived eigenstrain distributions, rather than using completely explicit FE analyses which require a high computation demand. The current paper aims to extend eigenstrain approach to estimate the residual stress generated in more complex geometric features where LSP is frequently applied in practice.

The paper presents the analysis of the effect of LSP applied close to straight/rounded free edges and also at leading edge geometries (in applications such as fan blades of aircraft engines). Initially, the residual stresses are determined by undertaking more detailed explicit FE analysis of the LSP process, and then we will proceed to develop an eigenstrain-based approximation to the configurations and assess its accuracy against the results from the explicit FE models. In order to model the residual stress using eigenstrain approach, the knowledge of distribution of eigenstrain is a prerequisite. The extent of any edge effects on the eigenstrain generated in the vicinity of a free edge is first investigated by considering an explicit FE model of a single laser pulse. Once the eigenstrain distribution in the vicinity of free edges has been determined, the residual stress distribution will be conveniently determined by introducing the eigenstrain as a misfit strain in a static/elastic FE model. Furthermore, using both completely explicit approach and eigenstrain approach we will investigate the effects due to a narrow untreated zone adjacent to the free edge which can 
occur in real-life LSP applications due to alignment difficulties and/or variations in the sacrificial aluminium tape applied around the edge. The paper also presents the eigenstrain/ explicit analyses of the residual stresses developed under a corner shot, a LSP pulse applied at an angle covering the edge with a reasonable overlap with the shots in the two flat surfaces, to counteract effects due to a narrow untreated zone adjacent to a free edge. Furthermore, using explicit FE analysis, it is demonstrated that the compressive surface stress developed in the vicinity of a rounded free edge is significantly higher than that generated at a straight edge.

When a laser pulse is fired at the surface, the surface layer (or the sacrificial tape) is vaporised, producing a rapidly-expanding plasma. A jet of water which simultaneously sprayed on the work surface during the application of LSP confines the rapidly expanding plasma $^{4}$. The effect of this is to generate a short duration high-pressure, shock (pressure) pulse in the work piece ${ }^{5}$. Transmission of the pressure pulse causes plastic deformation in the surface layers of the component and, after the pressure has decayed, the misfit between the plastically deformed material and surrounding elastic bulk generates a residual stress field. Usually, LSP treatment generates a surface compressive residual stress layer of 1-2 mm thick, and this is significantly thicker than that generated due to shot peening ${ }^{4}$. Since the laser parameters are more controllable than the critical parameters in shot peening, LSP can be used in specific design requirements with a high accuracy. However, it should be noted that a surface compression cannot be obtained without developing tension in the sub-surface of the component. In some applications this balancing tension can actually reduce the fatigue life ${ }^{3}$.

It is assumed that development of the residual stress due to LSP is mostly a mechanical process ${ }^{4}$. An explicit FE analysis is generally required to model the stresses caused by LSP. However, to obtain the stabilised residual stress distribution, the FE simulation needs to continue until the dynamic stresses caused by the laser pulse fully dissipate, and the solution 
is likely to be computationally costly, even if artificial material damping is introduced. This means that a completely explicit approach may be impractical, particularly since arrays of hundreds of LSP pulses are used in a practical peening application. The computational cost may be significantly reduced by using an eigenstrain approach. Previous work ${ }^{3}$ has shown that the plastic strains are usually stabilised within a relatively short time period (typically 1-2 $\mu$ s after the laser pulse ${ }^{1}$ ) and hence the stabilised plastic misfit strain (i.e. eigenstrain) can be conveniently extracted from an explicit FE simulation. The residual stress distribution can be calculated as the solution of the elastic FE model of the workpiece when the eigenstrain has been introduced as an initial misfit strain in a static FE model ${ }^{1}$ rather than seeking it directly. This approach has the benefit that the eigenstrain appears to be more characteristic of the process and it is largely independent of the component geometry whereas the residual stress field is influenced by the exact geometry of the work piece. By this we mean that the eigenstrain field is strongly dependent on the process and only weakly dependent on the component geometry, whereas the residual stress generated is influenced by both of these factors. The earlier work ${ }^{2}$ has also shown that the eigenstrain distribution produced by an array of LSP pulses in a single layer can be simply modelled as the same eigenstrain depth profile caused by a single shot but distributed over the area of the surface patch ${ }^{2}$. It was also shown $^{2}$ that the eigenstrain depth profile caused by multiple layers of LSP is largely unaffected by the size of the surface patch or by the amount of overlap between the subsequent layers. This enables modelling the residual stress caused by multiple layers of LSP shots by using the knowledge of eigenstrain depth profile under a simple representative array. In our previous work $^{1}$ the results determined from the eigenstrain model were validated against equivalent explicit FE simulations and also against experimental results of surface deformation $^{1}$, and with the plastic strain depth profile measured using diffraction peak broadening in a neutron diffraction experiment ${ }^{2}$. 
The previous work $^{1-2}$ was focused on the development of an accurate eigenstrain model to characterise the fundamental mechanics that generates residual stresses due to LSP. It was therefore appropriate to model simple geometries to ensure that the underlying physical behaviour was not obscured by geometrical factors. Hence, this earlier work concentrates on LSP treatment of surface patches in the centre of large flat surfaces. The analyses illustrate that the eigenstrain distribution generated under a LSP pulse forming part of an array is substantially limited to the volume directly under the pulse. Furthermore, the principal directions of the eigenstrain coincide with those of the coordinate axes of the geometry; thus, the eigenstrain distribution can be conveniently incorporated in a suitable elastic FE model. However, the analysis of LSP in non-planar components (e.g. close to a straight or a curved edge) is more difficult since peening is often carried out in more than one direction. Under these conditions, the principal directions of the eigenstrain cannot easily be predicted and incorporation of the eigenstrain in a static FE model is a potentially challenging problem. As described previously, the current paper investigates the degree to which LSP treatments of complex geometric features, representing parts of structural components under conditions relevant to practical service applications, can be analysed by using the knowledge of eigenstrain depth profile generated under simple LSP arrays. The analyses suggest that the residual stress caused by LSP treatment in the practical geometries considered in the study can be reasonably well estimated using this approach. The results show that an interaction between the process and geometrical features is exists and shows that this can lead to unexpected results such as surface tensile stress.

\section{Analysis of LSP treatment of a right angle free edge}

In order to understand the effect of LSP in geometric features relevant to service applications, we will commence by analysing surface treatment applied close to a straight free edge. The specific example is shown in Figure $1 \mathrm{a}$ and consists of a $16 \times 8 \times 8 \mathrm{~mm}$ Ti-6Al-4V specimen, 
peened by two pulses (each $3 \times 3 \mathrm{~mm}$ in size; laser power density $(I)=9 \mathrm{GW} / \mathrm{cm}^{2}$; and laser pulse duration $\left(t_{L}\right)=18 \mathrm{~ns}$ ) on the two surfaces adjacent to the free edge. For simplicity it was assumed that the shots were applied at the centre of the block in the $x$ direction. Symmetry considerations therefore require only half of the block to be modelled (Figure 1a).

\section{Explicit finite element modelling}

Material model. Titanium alloy Ti-6Al-4V was chosen in the present study because of its use in compressor blades of aircraft engines. Typically, the level of work hardening in the material is not marked ${ }^{6}$, and an elastic/perfectly-plastic behaviour (yield stress =1000 MPa; Young's modulus $=110 \mathrm{GPa}$; Poisson's ratio $=0.3$; and density $=4400 \mathrm{~kg} / \mathrm{m}^{3}$ ) was assumed in the current analyses; this is satisfactory for the purpose of validating the approach ${ }^{1-3}$. The results of earlier analyses ${ }^{1-2}$ show that during LSP, material will subject to high strain rates ( $10^{6} \mathrm{~s}^{-1}$ ). Hence a degree of strain rate hardening might be expected. Although more sophisticated material models (e.g. Johnson-Cook material model which can include effects of strain and strain rate hardening) could easily be employed in the explicit FE simulation, there is always a difficulty in setting the hardening parameters correctly. Material data for Ti$6 \mathrm{Al}-4 \mathrm{~V}$ at the strain rates of $10^{6} \mathrm{~s}^{-1}$ is difficult to obtain, particularly under reverse loading conditions. It should also be noted that the Johnson-Cook model frequently only calibrated against test data for strain rates up to $10^{4} \mathrm{~s}^{-1}$, hence extrapolating the model to strain rates of the order of $10^{6} \mathrm{~s}^{-1}$ will give an additional source of uncertainty. Hence use of a more sophisticated material model is difficult to justify. In any case, even if strain rate hardening takes place, the high levels of yield stress are unlikely to be relevant, since the residual stress field is generated as elastic response of the specimen to the misfit strains caused by the plastic strain generated due to LSP. The literature on what happens during relaxation after a high strain rate event is incomplete, and, from a physical standpoint it would seem that strain rate hardening results from dislocation dynamics, which would not be reproduced in subsequent 
quasi-static loading. Hence strain rate hardening is quite distinct from strain hardening where the increased yield stress may be sustained on subsequent loading. Thus, a quasi-static value is likely to be reasonably representative of the situation at the end of the LSP process.

(a)

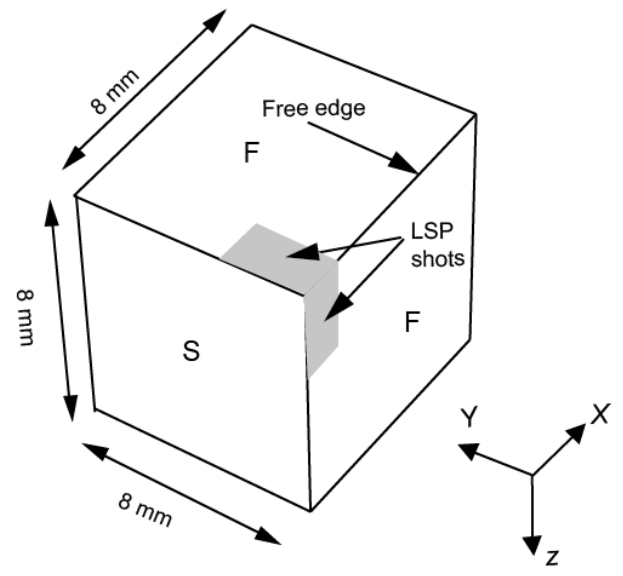

Figure 1a - An edge is treated from both the directions to achieve a desired residual stress $(\mathrm{F}=$ Free plane; $\mathrm{S}=$ Symmetry plane $)$ (b)

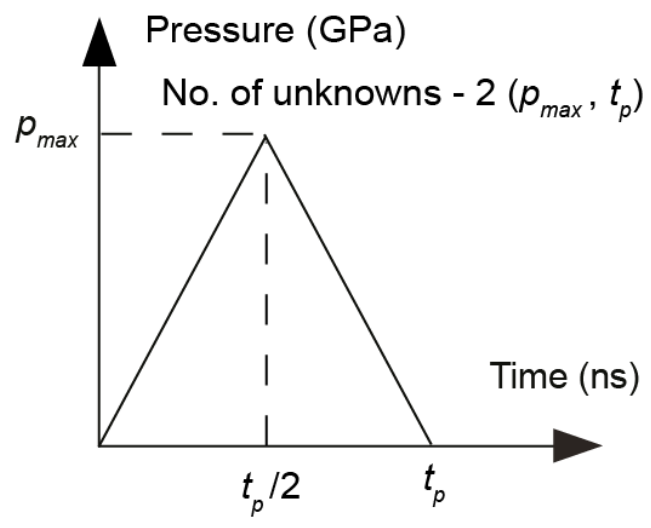

Figure 1b - Assumed variation of pressure with time, caused by application of a laser pulse

Characterisation of the pressure pulse. As explained in greater detail in the earlier papers ${ }^{1-2}$, the LSP process is modelled as a purely mechanical process resulting from the pressure induced by the plasma. Hence, in an explicit FE model, a pressure pulse is applied to the surface area underneath the laser shot. The pressure is treated as uniform over the area of the shot. Whilst this is probably an approximation, it is difficult to make a more sophisticated model without a detailed multi-physics model of the plasma generation process. It should also be considered that, in practice, to treat a surface region, multiple LSP shots are applied adjacent to each other with a small overlap ${ }^{7}$, and these small overlaps counteract any edge effects, thus, the pressure distribution developed under a LSP surface patch is largely uniform.

It remains to determine the time history of the pressure pulse applied. For simplicity, and because experimental results are again limited, a simple triangular variation of pressure with time was assumed (Figure 1b). The duration of the pulse is $t_{p}$ and the peak pressure, $p_{\max }$, is assumed to be generated at half the total duration of the pressure pulse, i.e. $p\left(t_{p} / 2\right)=p_{\max }$. 
The pressure pulse duration is normally assumed to be four to six times that of the laser pulse $^{8}$. Here a value of $t_{p}=100$ ns was used, which can be compared to the laser pulse duration, $t_{L}$, of $18 \mathrm{~ns}$. The final parameter needed is the peak pressure. This may be chosen by considering the energy transferred to the work piece by the pressure pulse and comparing this to the laser energy ${ }^{1}$. Typically, only $\sim 5 \%$ of the laser energy is transferred to the substrate ${ }^{7}$. In the current application (peak laser power density $=9 \mathrm{GW} / \mathrm{cm}^{2}$ and $t_{L}=18 \mathrm{~ns}$ ) the $p_{\max }$ in the simulation was adjusted so that $4.5 \%$ of the energy was transferred to the work piece ${ }^{1}$. This resulted in a peak pressure in the simulation of $6.7 \mathrm{GPa}$. These values are reasonably consistent with parameters experimentally determined in the literature for similar LSP systems, such as those reported by Fabbro et al. ${ }^{5}$ The assumed values for $p_{\max }$ and $t_{p}$ in the modelling have been validated to some extent in Achintha \& Nowell ${ }^{1}$ by comparing the results of the simulation with a simple experiment of comparing the experimental and predicted surface profiles obtained for the cases of single LSP shots.

\section{Residual stress distribution obtained from an explicit finite element model}

In order to provide a comparison with the eigenstrain approach, results are first generated from an equivalent explicit FE analysis. In the current study LS-DYNA (2007) ${ }^{9}$ was used for explicit FE simulations. The residual stress distribution was modelled by introducing two dynamic pressure loads $\left(p_{\max }=6.7 \mathrm{GPa}\right.$ and $t_{p}=100 \mathrm{~ns}$ ), representing the two LSP shots. In order to reduce the time taken for the elastic stress waves to decay, a degree of artificial material damping was turned on after the first pulse, once the initial plastic deformation was complete. This was then turned off before applying the subsequent pulse. Figure 2 shows the distribution of the stabilised residual stress components $\sigma_{x x}$ and $\sigma_{y y}$ in the vicinity of the free edge (It should be noted that the distribution of $\sigma_{z z}$ stress component in the specimen is not shown in Figure 2 since it is similar to the $\sigma_{y y}$ distribution because of the symmetry of $x z$ and $x y$ planes). As expected, no significant surface compressive stress is developed in the 
direction perpendicular to the free surface (e.g. $\sigma_{y y}$ ) because of the equilibrium requirements. It will be noted that there is very little difference in the $\sigma_{x x}$ stress distribution on the two peened faces, suggesting that the order of the two pulses is relatively unimportant and therefore that superposition of the individual effects may be considered appropriate. There is a degree of surface compression in the $x x$ direction on both free surfaces, with a small region of tension at the edge itself. The subsurface area of balancing tension is also clearly visible in Figure 2a. When LSP pulses are applied on the two surfaces that form a $90^{\circ}$ edge, a high misfit strain can be developed since the plastic strain caused by the second shot will be superposed on that already developed due to the first shot. Subsequently, a relatively high peak subsurface tension can be expected and the predicted value of $\sim 400 \mathrm{MPa}$ is comparable with the peak tension observed in flat samples ${ }^{2,3}$. Rather than undertaking a more detailed analysis of the results from the explicit FE simulation, we will proceed to develop an eigenstrain-based approximation to the configuration and assess its accuracy.
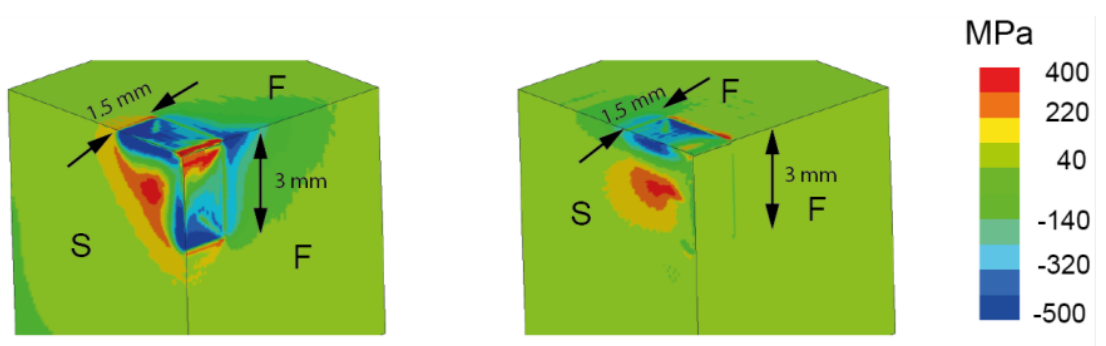

(a)

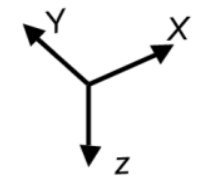

(b)

Figure 2 - Stabilised residual stress distribution determined from a completely explicit FE

$$
\text { simulation }\left(\text { a) } \sigma_{x x} \quad \text { (b) } \sigma_{y y} \quad(\mathrm{~F}=\text { Free plane, } S=\text { Symmetry plane })\right.
$$

\section{Determination of the eigenstrain from an explicit finite element model}

We start by noting our earlier result ${ }^{1-2}$ that the eigenstrain distribution generated due to a laser shot applied in the central region of a large flat surface of a thick specimen is approximately uniform in the in-plane directions and varies only in the direction perpendicular to the surface. However, this does not imply that the residual stress distribution is uniform (see for example Brockman et al. ${ }^{10}$ or Dorman et al. ${ }^{11}$ ). Indeed, one of the advantages of the eigenstrain 
approach is that the eigenstrain introduced by a process is often much simpler than the resulting stress field. However, with the current geometry of an LSP shot applied adjacent to a free edge of a specimen, the free edge is unlikely to provide the same constraint against plastic deformation as that provided by the material in other directions. To characterise the residual stress field using the eigenstrain approach, an estimate of eigenstrain distribution present in the specimen must be obtained. The extent of any edge effects on the eigenstrain generated must be investigated, and we therefore start by considering an explicit FE model of a single laser pulse applied at different distances from the free edge.

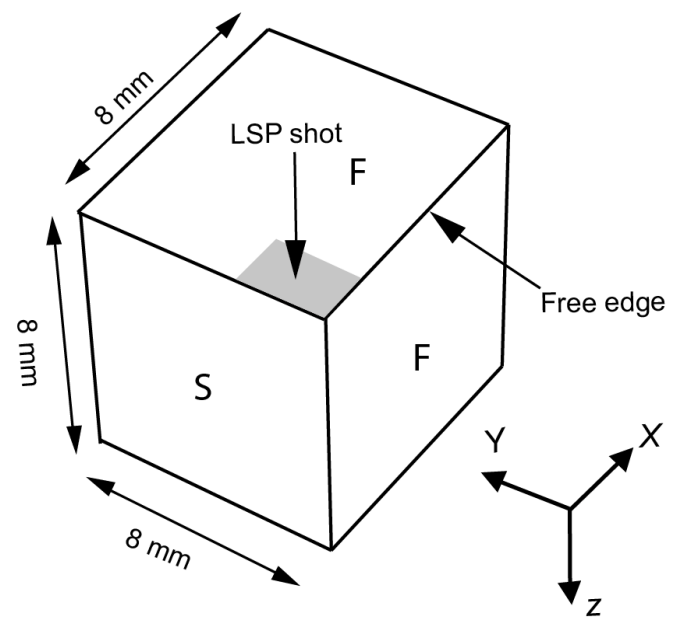

Figure 3 - A single LSP shot pulsed adjacent to a straight free edge

$$
(\mathrm{F}=\text { Free plane } \mathrm{S}=\text { Symmetry plane })
$$

\section{Edge effects in the eigenstrain distribution}

As an example to study of the effect of a free edge, the aforementioned $16 \times 8 \times 8 \mathrm{~mm}$ Ti- $6 \mathrm{Al}-$ $4 \mathrm{~V}$ block, is treated by a single laser pulse of the same laser system previously modelled. For simplicity it was assumed that the laser shot was applied at the centre of the block in the $x$ direction. Symmetry considerations therefore require only half of the block to be modelled (Figure 3). A dynamic pressure pulse with $p_{\max }=6.7 \mathrm{GPa}$ and $t_{p}=100 \mathrm{~ns}$ underneath the LSP pulse was modelled in a explicit FE analysis. The resultant eigenstrain distribution determined from the explicit FE simulation is shown in Figure 4a. To facilitate the understanding of the influence of free edge on the eigenstrain distribution generated it is compared with that caused by a LSP pulse applied well away from the edge where the influence of free edge is not 
significant (Figure 4b). For the edge pulse, the results show an influence of the free edge on the eigenstrain distribution generated, in particular, within about $0.5 \mathrm{~mm}$ from the free edge and in the vicinity of the surface (up to a depth of $\sim 0.5 \mathrm{~mm}$ ). However, the results shows that this effect is very localised and approximately $0.5 \mathrm{~mm}$ away from the free edge the two eigenstrain distributions are very similar. Figure $4 \mathrm{c}$ compares the variation of eigenstrain with depth $(z)$ at the centre point of the two LSP shots; it can be seen that they are very similar. Hence, away from the immediate vicinity of the edge, the eigenstrain may be assumed to be the same as that generated by a pulse applied in the centre point of a large work piece. However, it should be noted that although the eigenstrain depth profiles are similar, the resultant residual stress distributions in the two specimens are not the same. The residual stress field arises as elastic response of the specimen for the eigenstrain distribution present in the specimen. As it is shown in the latter part of the current paper, the elastic response of the component strongly depends on the component geometry and the constraint provided by the elastic material around the plastically deformed zone under the LSP pulse. For the edge pulse, the free edge is unlikely to provide the same constraint against plastic deformation as that provided by the material in other directions and hence a different residual stress than that developed under a central LSP pulse applied well away from the edge is developed. It is the main objective of the current paper is to demonstrate that the eigenstrain method may provide an attractive method for investigating different peening schemes. The eigenstrain distribution is a more characteristic of the LSP process and hence to determine residual stress generated due to the treatment around free edges of structural components all that is required is knowledge of eigenstrain depth profile caused by the process to a single patch on a flat plate.

\section{Approximate representation of eigenstrain caused by an edge pulse}

Since the effect of a free edge is limited to a very narrow region, it is possible to make a first approximation to the eigenstrain introduced by an edge pulse by assuming it to be uniform (in 
$x$ and $y$ ). This will allow a first order investigation of the stresses close to the edge, which we expect will be more influenced by the boundary conditions than will the eigenstrain. Whilst a more complex analysis with non-uniform eigenstrain might be possible, it is important to realise that the physics of the shock wave generation might also be affected by the edge, and the assumption of a uniform pressure pulse might also need to be called into question.

(a)

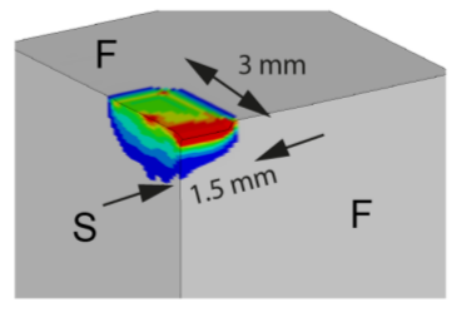

(c)

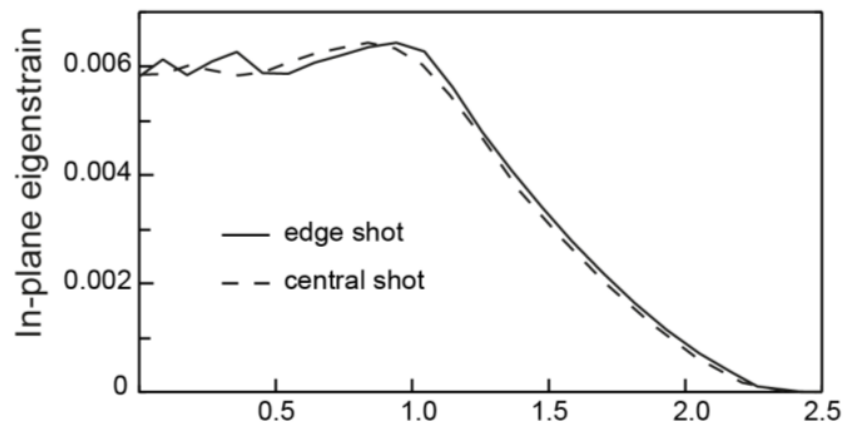

Depth from the surface, $z(\mathrm{~mm})$ (b)
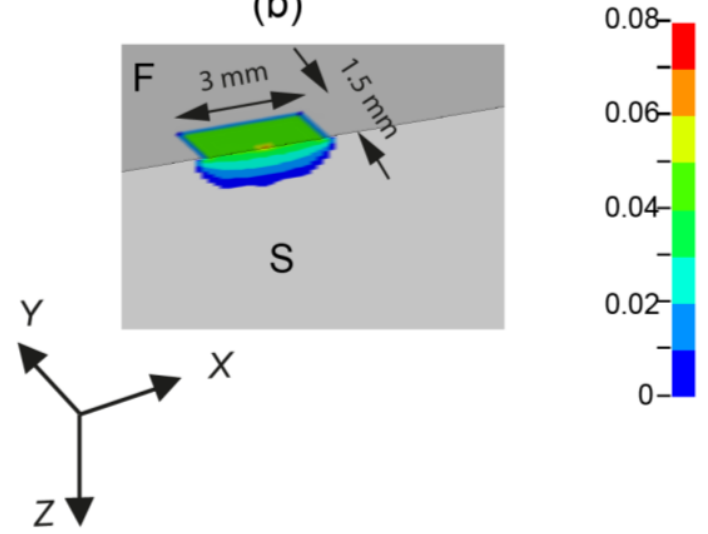

Figure 4 - Eigenstrain distribution under an

(a) edge pulse

(b) central pulse

(c) eigenstrain depth profile at the centre point of an edge pulse and a central pulse

$$
(\mathrm{F}=\text { Free plane } \quad \mathrm{S}=\text { Symmetry plane })
$$

The validity of the uniform eigenstrain assumption close to a free edge may be investigated further by investigating the effect of a small offset between the pulse and the edge. The effect of pulses with offsets of $0.15,0.30 \mathrm{~mm}$ (i.e. $5 \%$ and $10 \%$ of the size of the pulse) respectively (Figure 5) applied on the same specimen (Figure 1a) is studied below. Explicit FE simulations were carried out by applying the correct dynamic pressure load over the area corresponding to the chosen offset. Figures $6 a$ and $6 b$ show the eigenstrain distributions obtained from these analyses for each offset. It can be seen from the figures that the eigenstrain distribution is mostly uniform in $x$ and $y$ directions and it is very similar to that produced by a LSP pulse 
applied away from the edge. Figure $6 c$ compares the eigenstrain depth profile at the centre of the LSP shots with 0.15 and $0.30 \mathrm{~mm}$ offsets with that for a pulse applied remote from the edge. The results suggest that the effect of a free edge is not significant if the pressure is developed with a small offset from the edge. In such circumstances it is reasonable to assume a uniform eigenstrain distribution. This allows modelling the residual stress field produced by a LSP shot applied close to a free edge in the usual way ${ }^{1-2}$ by incorporating a simplified form of the eigenstrain distribution in a FE model.

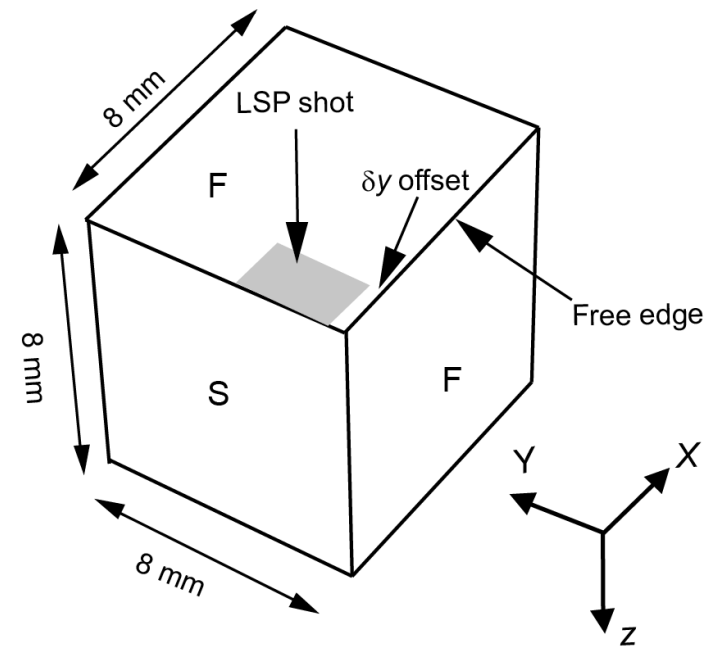

Figure 5 - A single LSP pulse with an offset from the free edge

$$
(\mathrm{F}=\text { Free plane } \quad \mathrm{S}=\text { Symmetry plane })
$$

\section{Eigenstrain modelling of two LSP pulses along a free edge}

The above results show that a first approximation to the eigenstrain distribution caused by an edge pulse may be achieved without taking explicit account of the edge effects on the form of the eigenstrain. Using this approach the effect of applying two LSP pulses, one on each of the two perpendicular surfaces along a free edge can be approximated by the superposition of the eigenstrain caused by individual pulses. To demonstrate this approach, the results of the same Ti-6Al-4V specimen (Figure 1a) $(16 \times 8 \times 8 \mathrm{~mm})$ when peened by two shots will be discussed.

\section{Superposition of eigenstrain distributions}

When LSP pulses are applied on the two surfaces that form the edge, a relatively complex 
eigenstrain distribution may be developed in the workpiece, and the eigenstrain caused by the second shot may be influenced by that present as a result of the first. However, the simplest approach is to postulate that superposition may be applied. It should be appreciated that the assumption of an elastic / perfectly plastic material model may hinder modelling of the exact eigenstrain distribution; however, it is the main objective of the current paper is to demonstrate that the eigenstrain method may provide an attractive simplified method for investigating residual stresses developed in the complex geometric features. In order to assess the appropriateness of the assumption the results of eigenstrain-based analyses may be compared with those obtained with an explicit analysis of the two shots (Figure 2).

(a)

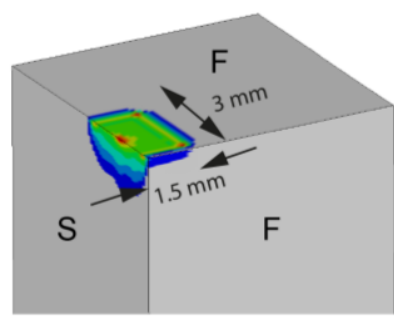

(b)
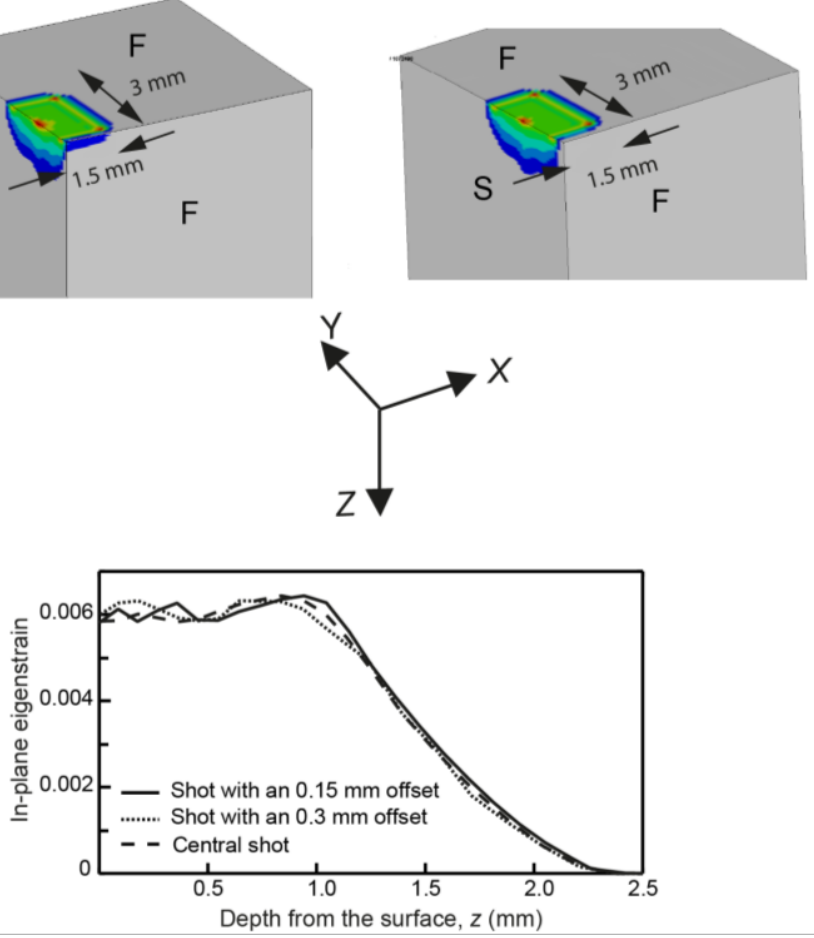

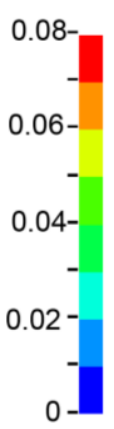

Figure 6 - Eigenstrain distribution under an LSP shot with an offset of

(a) $0.15 \mathrm{~mm}$

(b) $0.3 \mathrm{~mm}$ from the free edge $\quad$ (c) eigenstrain depth profile at the centre of an edge shot and that at a central shot $(\mathrm{F}=$ Free plane $\mathrm{S}=$ Symmetry plane $)$

Figure 7 (a), (b) and (c) show the three principal strain components of the eigenstrain distribution $\left(\varepsilon_{x x}^{p}, \varepsilon_{y y}^{p}\right.$ and $\varepsilon_{z z}^{p}$ respectively) determined by superposing the respective eigenstrains caused by the two pulses. Figure 7(d) shows the comparison between the depth 
profiles of the principal components of eigenstrain at the centre of the first pulse and those after the superposition of the eigenstrain generated due to the second pulse. By applying three principal components of the final eigenstrain in a static FE model (ABAQUS/Standard ${ }^{12}$ was used in the present study) the residual stress field can be determined. In order to introduce the correct eigenstrain at each point within the workpiece, this is modelled by specifying anisotropic thermal expansion coefficients that vary with position, together with a uniform (unit) temperature rise ${ }^{1}$.

(a)

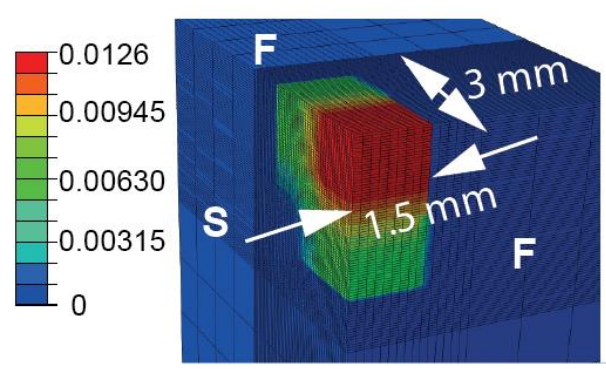

(c)

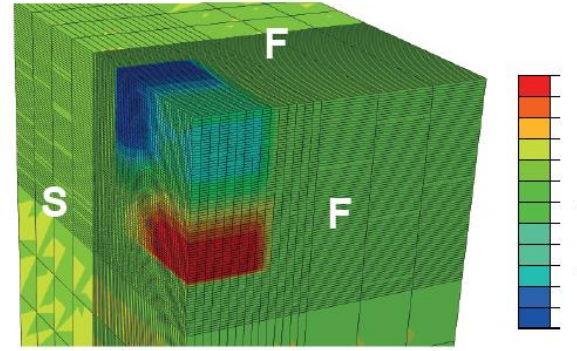

(b)

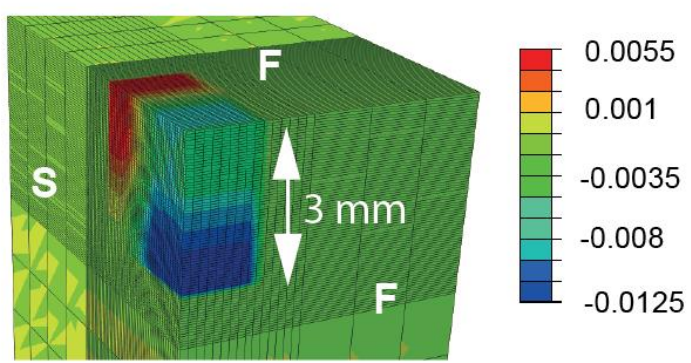

(d)

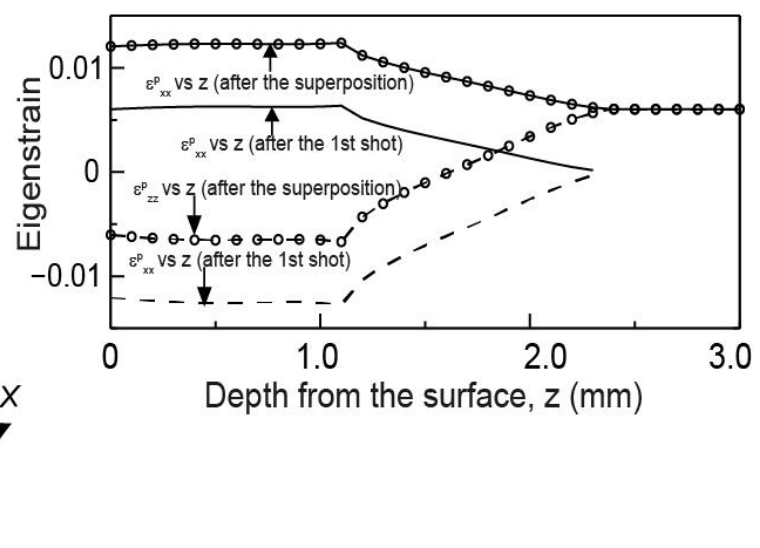

Figure 7 -(a) $\varepsilon_{x x}^{p}$ (b) $\varepsilon_{y y}^{p}$ (c) $\varepsilon_{z z}^{p}$ principal components of the eigenstrain (d) eigenstrain depth profile at the centre of the pulse after the $1^{\text {st }}$ pulse and after the $2^{\text {nd }}$ pulse

$$
(\mathrm{F}=\text { Free plane } \quad \mathrm{S}=\text { Symmetry plane })
$$

\section{Comparison of residual stress distributions from explicit and eigenstrain models}

Figure 8 shows the results of residual stress components $\sigma_{x x}$ and $\sigma_{y y}$ close to the free edge obtained from a static FE model after applying the eigenstrain distribution described above. This can be compared with the results determined from an equivalent wholly explicit FE analysis (Figure 2). For instance, Figure 8b compares the variation of $\sigma_{x x}$ stress component 
obtained from the eigenstrain method and from the fully explicit analysis. The figure shows the variation $\sigma_{x x}$ stress along a $45^{0}$ diagonal direction starting from the centre of the pulse on xy surface (i.e. $\sigma_{x x}$ along the line AA in Figure 8a). The comparison shows that the two sets of results are consistent. However, it should be appreciated that the eigenstrain model predicts slightly higher peak tension than the explicit FE approach. Furthermore, the small tension region along the free edge (Figure 2a) itself is not reproduced in the eigenstrain model because of the approximation of the eigenstrain with a uniform distribution in $x$ and $y$.
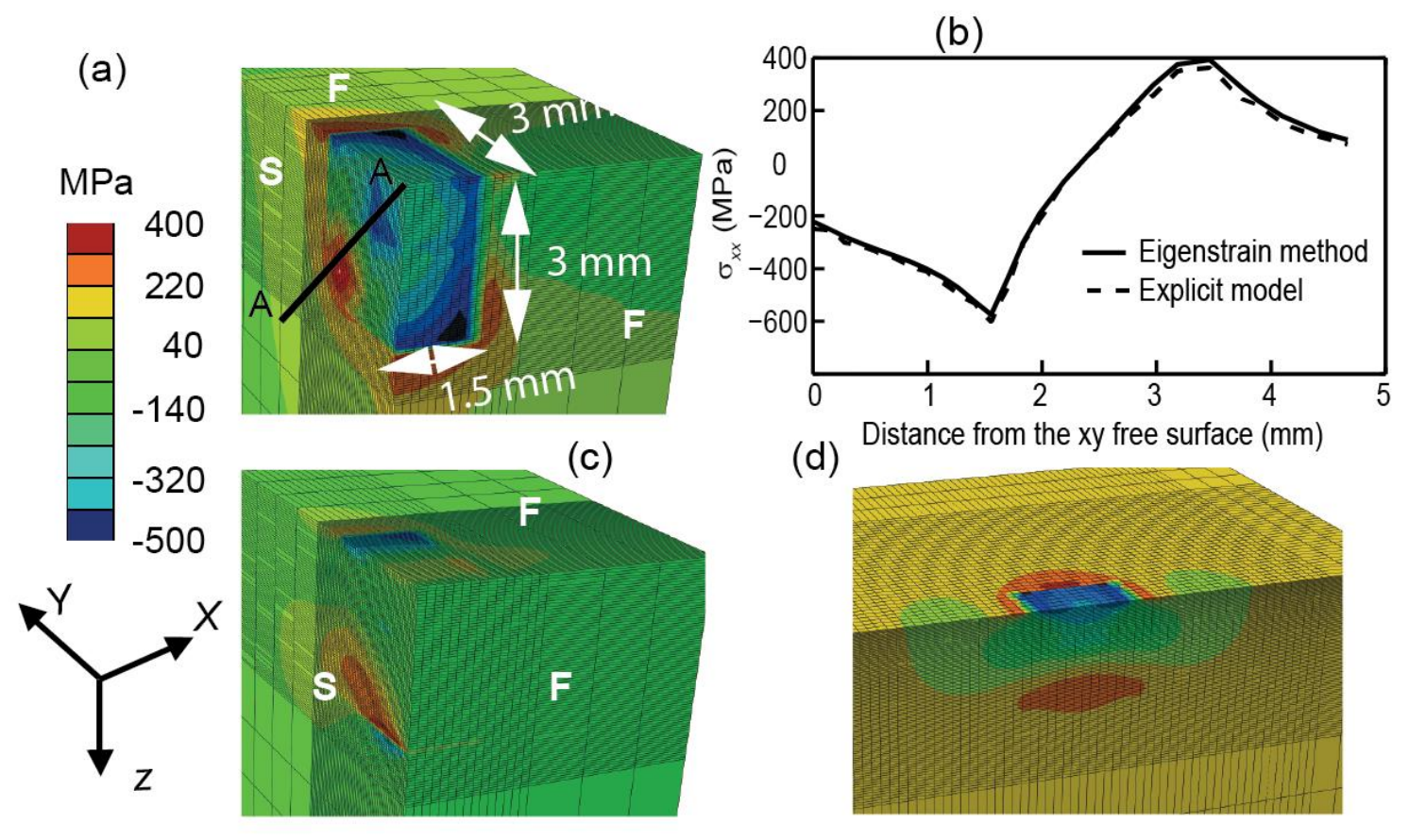

Figure 8 - Residual stress distribution: (a) $\sigma_{x x}$ stress at the edge (from eigenstrain model) (b) comparison between the $\sigma_{x x}$ stress component predicted from the eigenstrain method and from explicit analysis (c) $\sigma_{y y}$ stress at the edge (eigenstrain model) (d) $\sigma_{x x}\left(=\sigma_{y y}\right)$ under a central LSP patch (eigenstrain model $) \quad(F=$ Free plane $\quad S=$ Symmetry plane $)$

The residual stress component in the direction along the free edge $\left(\sigma_{x x}\right)$ (Figure 8a) is often the most important in design, as the $y y$ and $z z$ components will be close to zero by virtue of the free surfaces. To facilitate the understanding of the influence of the free edge, the results are compared with the residual stress distribution due to an equivalent LSP shot at the central region of the top surface of the same specimen (Figure 8d) (only a half of the specimen is 
shown in Figure $8 \mathrm{~d}$ and because of symmetry $\sigma_{y y}=\sigma_{x x}$ ). It is clear from Figure $8 \mathrm{a}$ that at an edge the surface compressive stress developed is not as marked as that produced by the same pulse remote from an edge ( $250 \mathrm{MPa}$ in comparison to $\sim 560 \mathrm{MPa}$ generated by a central pulse). Since the eigenstrain distributions are similar, the difference in the residual stress generated in the two specimens show the effect of the geometry on the constraint provided. Under a central shot, the residual stress is mostly uniform in the plane parallel to the surface, whereas in the vicinity of a free edge the stress field generated is non-uniform. However, the compressive stress gradually increases away from the surface and the peak compressive stress is $\sim 560 \mathrm{MPa}$, and occurs at $y=z \sim 1.1 \mathrm{~mm}$, which compares well with that under a central pulse $(z \sim 1.1 \mathrm{~mm})$. Similarly, the peak sub-surface tensile stress is $\sim 380 \mathrm{MPa}$ for an edge pulse, about twice the magnitude of that under a central pulse ( 190 MPa). However, the location of the tensile maximum $(y=z \sim 2.35 \mathrm{~mm})$ compares well with that for a central shot $(z \sim 2.35 \mathrm{~mm})$. For each geometry, the peak tension occurs at same location, approximately where the applied eigenstrain distribution ends. The transition between the misfit in the plastically deformed region and the elastic bulk means that the peak tension is likely to occur at the end of the eigenstrain distribution. In practice this seems to be true for a range of problems of this nature: the model prediction for the depth at which the peak tension occurs matches well with that found in experiments ${ }^{1,2}$.

As noted earlier, it can be shown by example ${ }^{2}$ that the eigenstrain depth profile caused by a given laser peen is largely independent of the component geometry, the time interval between subsequent LSP pulses in an array, or the small overlap between adjacent pulses in a surface patch. Thus, the knowledge of eigenstrain depth profile determined from a single explicit FE analysis, the residual stress introduced due to more complex practical LSP treatments can be modelled in a simplified manner, by applying the eigenstrain depth profile over an appropriate volume in a static FE model. For instance, Figures $9 \mathrm{a}$ and $9 \mathrm{~b}$ compare the resultant 
eigenstrain distribution extracted from explicit FE simulations of the specimen described above, (a) if the two shots were applied simultaneously and (b) after allowing the residual stress due to the first pulse to stabilise. Similarly, Figure 9c shows the variation of $\sigma_{\mathrm{xx}}$ at a point distance $0.5 \mathrm{~mm}$ away from the free edge with depth $\mathrm{z}$ from the surface. The results show that the residual stress depth profiles are very similar in the two cases. The figure shows that the eigenstrain is largely independent of the time interval between the two pulses. This understanding will be exploited in the remainder of the paper.

(a)
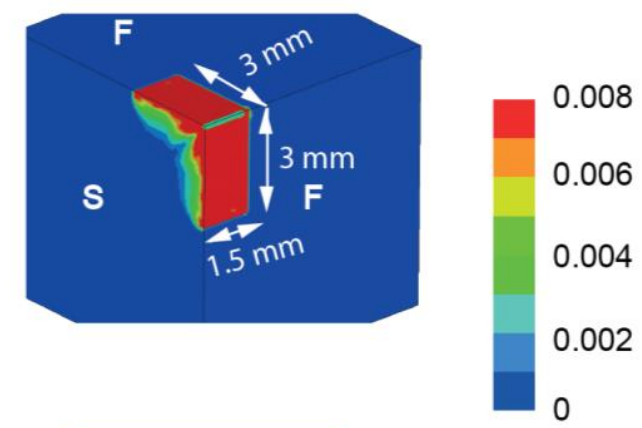

(b)
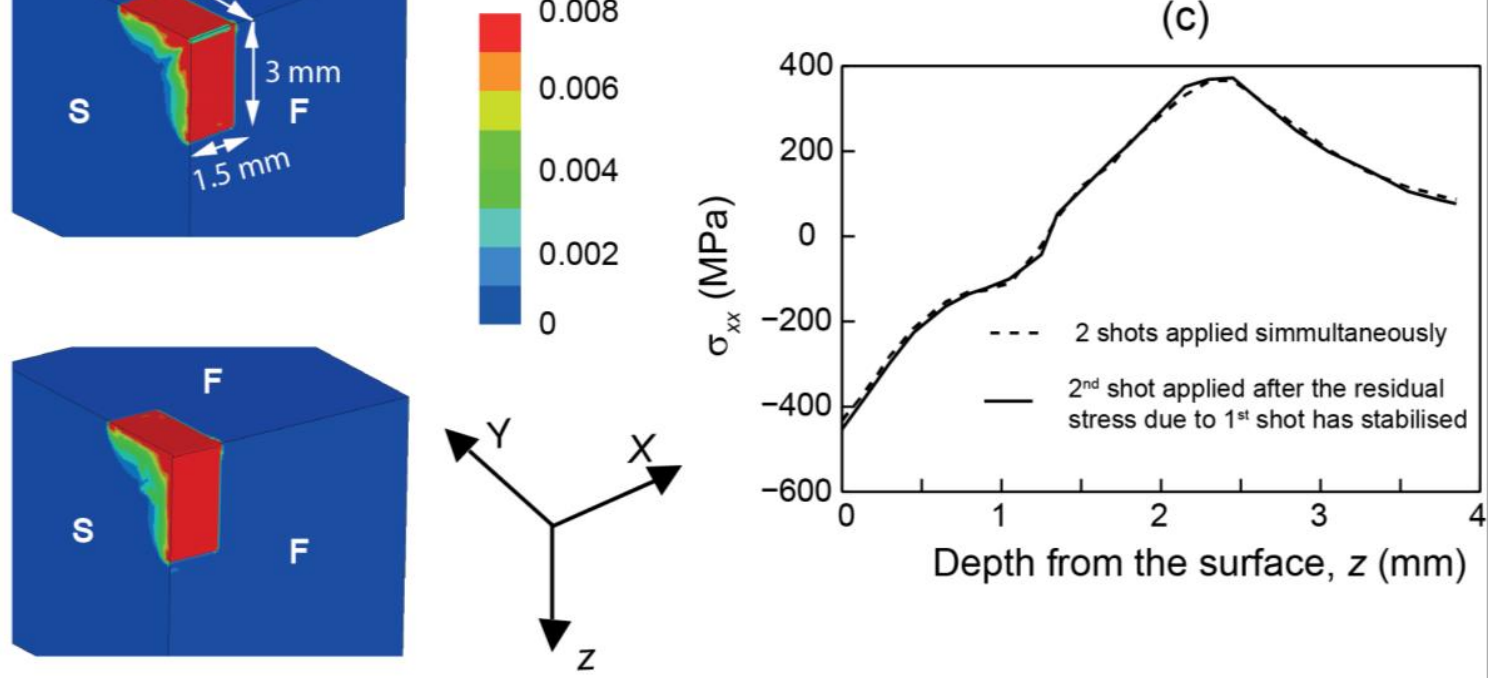

Figure 9 - Explicit FE results for the eigenstrain distribution (a) if the two shots were applied simultaneously (b) if the second shot was applied after the residual stress field due to the first pulse had stabilised (c) Residual stress depth profile at a point $0.5 \mathrm{~mm}$ from the free edge $(\mathrm{F}=$ Free plane $\mathrm{S}=$ Symmetric plane $)$

Eigenstrain modelling of the residual stress generated in the vicinity of a free edge due to a strip of LSP pulses

The results of previous analysis of two LSP pulses suggest that the residual stress field generated in the vicinity of an edge can be accurately determined from the eigenstrain approach by simply using the superposition of the eigenstrain caused by each shot. The analysis is extended now to analyse the effect due to long and $9 \mathrm{~mm}$ wide strips (on each of the surfaces) of LSP shots extending along the edge, representing a practical LSP treatment. 
We will discuss the results of a $80 \times 40 \times 30 \mathrm{~mm}$ Ti-6Al-4V specimen, treated with LSP strips of 9 and $27 \mathrm{~mm}$ long (each individual shot is $3 \times 3 \mathrm{~mm} ; I=9 \mathrm{GW} / \mathrm{cm}^{2} ; p_{\max }=6.7 \mathrm{GPa} ; t_{p}=$ $100 \mathrm{~ns}$ ) (Figure 10a). For simplicity, it was assumed that the pulses are applied at the centre of the edge and with no overlap between adjacent shots. The residual stress distribution is modelled by imposing eigenstrain depth profile (Figure 4c) over the required volume in static FE models.

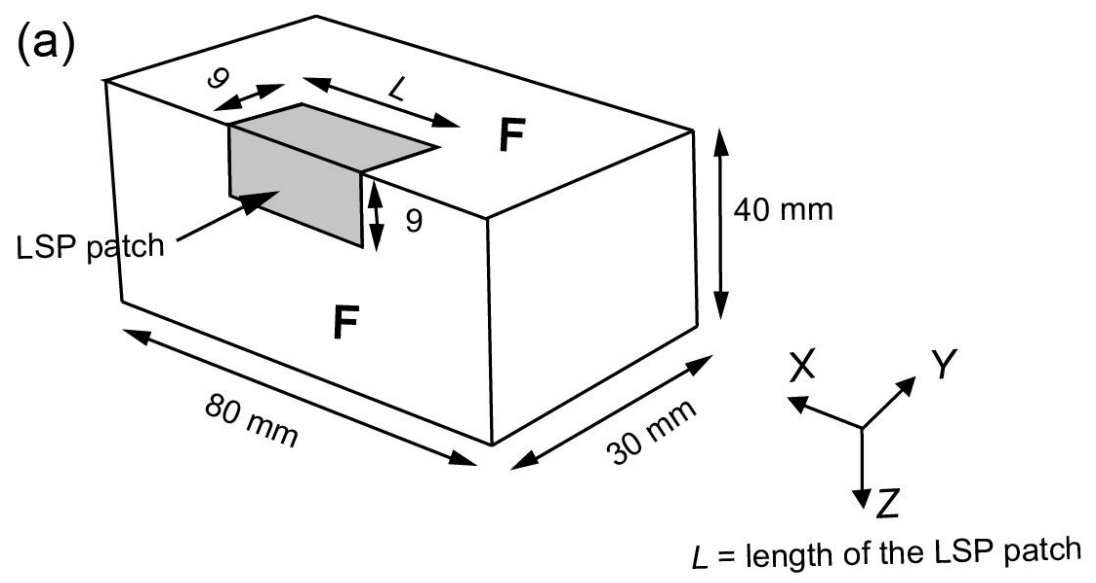

(b)

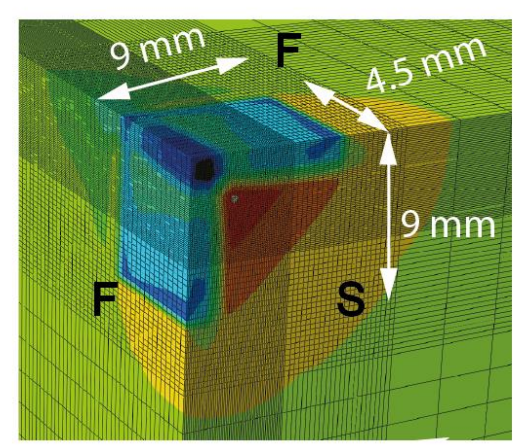

(c)

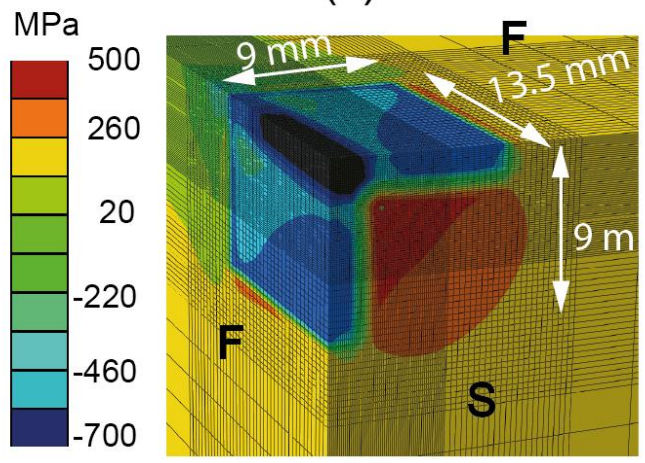

Figure 10 - (a) Treatment of a straight edge using an LSP strip $\sigma_{x x}$ stress distribution under the (b) short $(L=9 \mathrm{~mm})$ strip (c) long $(L=27 \mathrm{~mm})$ strip ( $\mathrm{F}=$ Free plane $\mathrm{S}=$ Symmetry plane)

Figures $10 \mathrm{~b}$ and $10 \mathrm{c}$ show the $\sigma_{x x}$ stress developed underneath the short and the long strip respectively. The results show that there is largely a uniform surface compressive stress, with a narrow zone of relatively high magnitude compressive stress adjacent to the free edge, developed underneath the long patch. This high stress close to the edge is attributed to the high eigenstrain component, $\varepsilon_{x x}^{p}$, generated by the two shots pulsed on the two free surfaces 
(Figure $7 \mathrm{a})$. As expected, the long patch produces a higher surface compression ( $650 \mathrm{MPa})$ than under the shorter strip $(\sim 500 \mathrm{MPa})$. It should be appreciated that the residual stress is a stress field that exists in the absence of external loading. Although the eigenstrain depth profiles are identical under the two patches of $9 \mathrm{~mm}$ and $27 \mathrm{~mm}$ long, there are essentially distributed over different volume of the component. Thus, the response of the component for these misfit strain (eigenstrain) distributions can be different, and in this case results in two different residual stress distributions. However, irrespective of the length of the surface patch, a peak subsurface tension of $\sim 380 \mathrm{MPa}$ develops at a depth of $\sim 2.5 \mathrm{~mm}$ from each surface. The depth of the compressive stress layer is $\sim 1.8 \mathrm{~mm}$ (from each surface) and this is largely independent of the length of the surface patch. The results suggest that a desirable surface compressive stress can be achieved at a free edge by applying an LSP strip along the edge. The eigenstrain method enables modelling residual stress in practical structural components by using the knowledge of the eigenstrain depth profile causes by the LSP system.

\section{Eigenstrain modelling of a partially-treated edge}

All the analyses described above were based on the assumption that an edge pulse is applied perpendicular to the work surface covering the whole area adjacent to the free edge of the specimen. However, in practice it is difficult to treat the vicinity of the free edge uniformly because of alignment difficulties or variations in the application of sacrificial aluminium tape applied around the edge. In such circumstances, it is possible that a narrow zone adjacent to the free edge remains untreated or experiences an eigenstrain of lower magnitude than elsewhere under the pulse. It should be appreciated that the problem investigated here is different to the earlier 'edge effect' investigation, which examined the non-uniformity of the eigenstrain distribution generated if a laser pulse is applied right along the free edge. To examine these possible effects results of the same $16 \times 8 \times 8 \mathrm{~mm}$ specimen (Figure 1a), treated by two LSP shots (each $3 \times 3 \mathrm{~mm}$ with the same laser system $\left(I=9 \mathrm{GW} / \mathrm{cm}^{2}, p_{\max }=6.7 \mathrm{MPa}, t_{p}\right.$ 
$=100 \mathrm{~ns})$ ). It is assumed that a narrow strip of $0.3 \mathrm{~mm}$ wide (i.e. one tenth of width of a LSP pulse) remains untreated adjacent to the free edge on each work surface (Figure 11a).

(a)
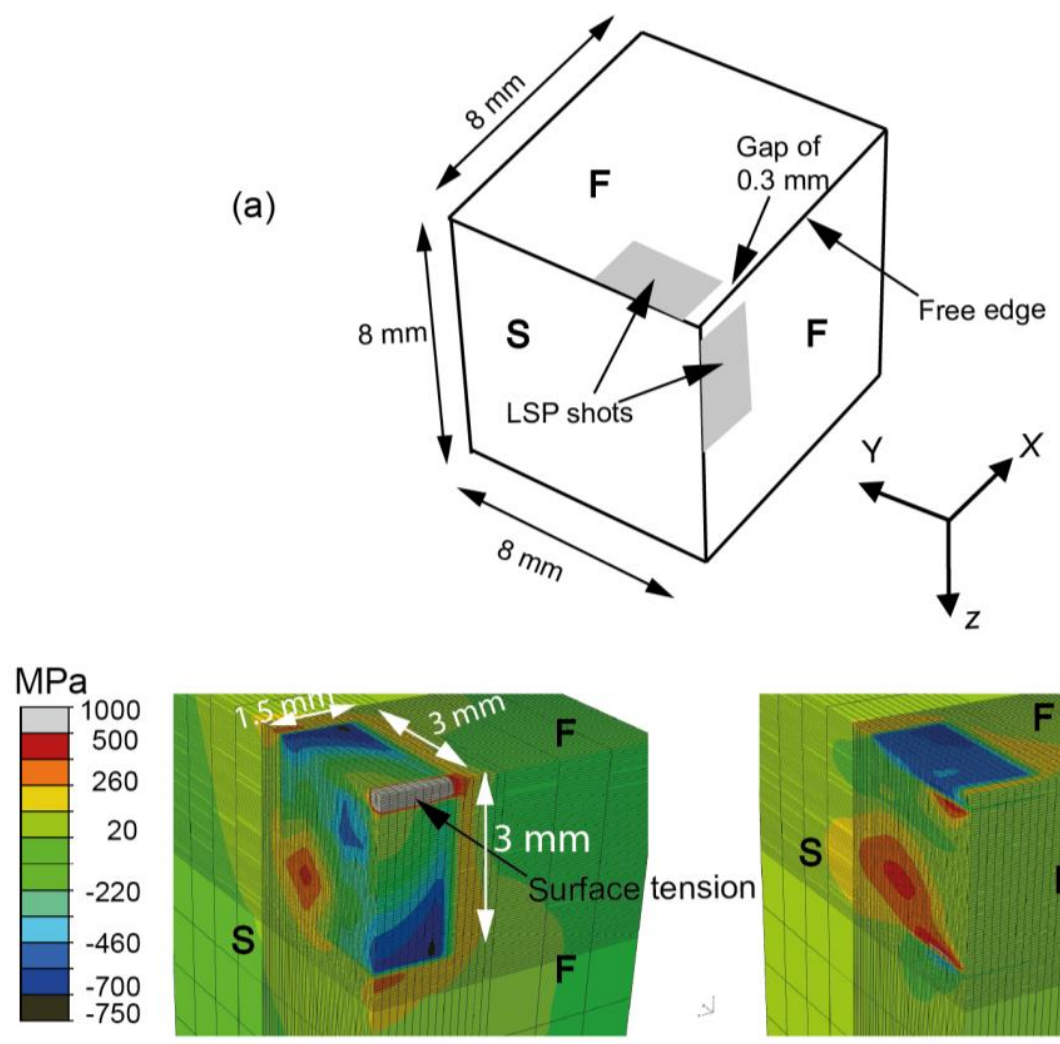

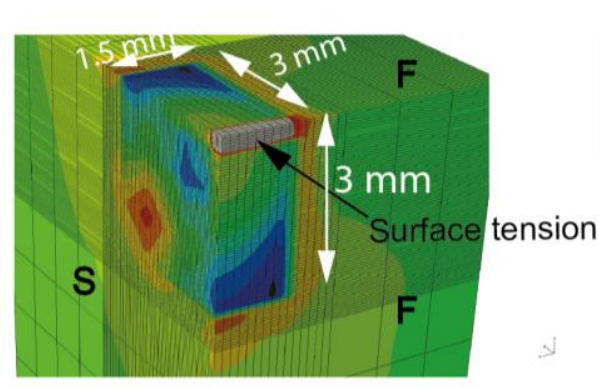

(b)

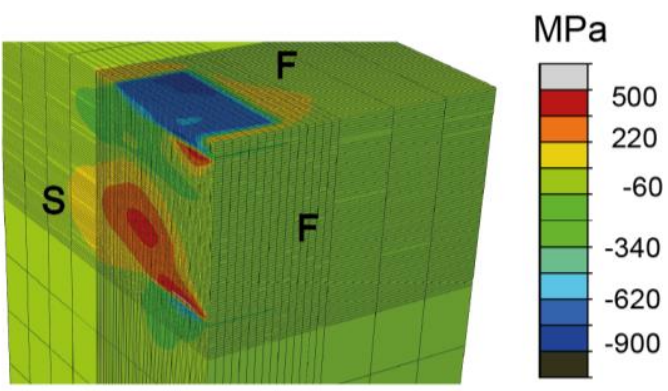

(c)

Figure 11 - (a) A narrow untreated strip adjacent to the free edge.

$$
\begin{array}{lll}
\text { Residual stress distribution } & \text { (b) } \sigma_{x x} & \text { (c) } \sigma_{y y}
\end{array}
$$$$
(\mathrm{F}=\text { Free plane } \quad \mathrm{S}=\text { symmetric plane })
$$

This problem is analysed by simply incorporating the correct eigenstrain depth profile (Figure 4c) over the appropriate volume within the static FE model. Figures $11 \mathrm{~b}$ and $11 \mathrm{c}$ show the stress $\sigma_{x x}$ and $\sigma_{y y}$ stress components close to the free edge respectively. The results illustrate that the main residual stress field here is similar to that generated by an equivalent array of LSP pulses that covers the edge fully (Figures 8a and 8c). However, the results suggest that a high $\sigma_{x x}$ surface tension of magnitude $\sim 900 \mathrm{MPa}$ is developed close to the vicinity of free edge. Although this stress is highly localised, the surface tension is significantly higher than the subsurface peak tension ( 380 MPa) and in these circumstances, the LSP treatment may reduce the fatigue life of the component. Since the eigenstrain depth profiles are the same, comparison of these results with the previous results for a fully-treated 
edge (Figures 8a and 8c) highlights the significant influence of the precise location of the LSP patch. This effect is due to the variation of the constraint provided by the elastic bulk material in response to the plastic strain caused by LSP.

\section{Corner shots used to treat a straight edge}

The results described above illustrate that if the edge is not fully treated by the LSP, a significant surface tension may develop in the vicinity, potentially reducing the strength of the component. Even if an edge is fully-treated, the explicit results in Figure 2 suggest that a nonuniform eigenstrain may cause tension in this region. One way to counteract this edge effect is by introducing a corner shot where a LSP pulse is applied at an angle covering the edge, and with a reasonable overlap with the shots in the two flat surfaces (Figure 12a).

\section{Explicit FE modelling of the residual stress due to a corner shot}

In order to determine the effect of a corner shot we will analyse the standard specimen (Figure 1a) for a LSP shot applied at an angle of $45^{\circ}$ to the two surfaces (Figure 12a). The orientation of the pulse means that the laser beam will now be spread over a larger area of the specimen than with a normal pulse. In such circumstances, the intensity of the LSP pulse $(I)$ and $p_{\max }$ of the resulting shock wave will be smaller than those with a normal shot. We assume a $I$ of

$9 \sqrt{2} \mathrm{GW} / \mathrm{cm}^{2}$ for the corner shot since this will result in a pressure pulse with the same $p_{\max }$ as a normal shot $(6.7 \mathrm{GPa})$. As shown below, this will allow the use of the knowledge of the eigenstrain depth profile generated under a normal shot (Figure 4c) to be used in the analysis of residual stress generated.

\section{Eigenstrain modelling of the residual stress due to a corner shot}

The exact distribution of eigenstrain caused by a corner shot is difficult to determine and again we will use the uniform eigenstrain approximation. Earlier analysis shows that 
eigenstrain caused by an array of LSP shots can be modelled by the superposition of that due to each shot in the array, it is appropriate to analyse the effect of a corner shot by analysing initially as two equivalent shots applied on the two adjacent flat surfaces. It should also be noted that in this analysis the size of the corner pulse was chosen so that the pulse treats a $3 \times 3$ $\mathrm{mm}$ projected area on each surface since this effectively the same situation as the previously analysed problem of two LSP pulses, one after other, on each of the two flat surfaces. Figures $12 \mathrm{~b}$ and $12 \mathrm{c}$ provide comparison between the residual stress distribution obtained from the eigenstrain analysis and that from an equivalent explicit FE analysis, respectively. The figure shows that results from the two methods compare well. A surface compressive stress layer of very similar magnitude and depth is developed in each case. A peak tension of $\sim 380 \mathrm{MPa}$ is developed at $y=z \sim 2.3 \mathrm{~mm}$ for each case. The results suggest that a desirable surface compressive stress can be achieved at a free edge by applying corner LSP shots; the eigenstrain approach provides an attractive simplified method to model the residual stress generated.

(a)

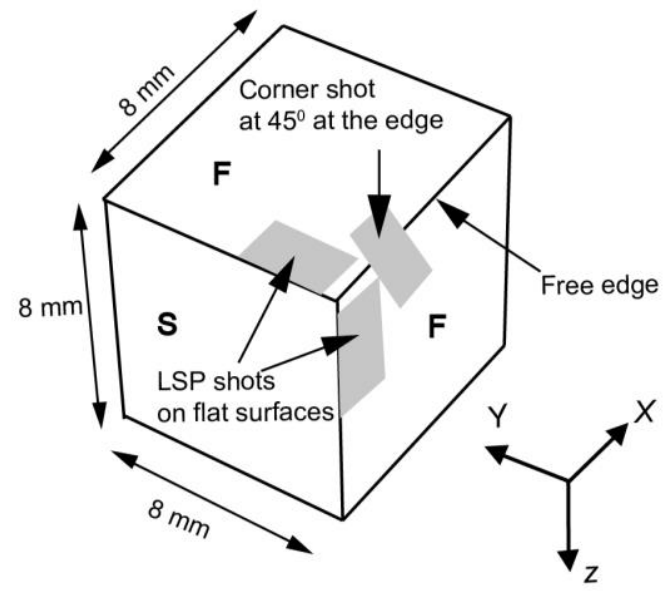

(b)

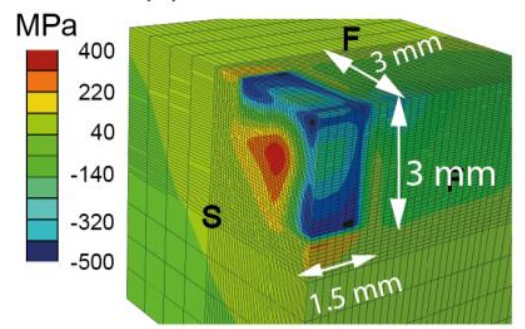

(c)

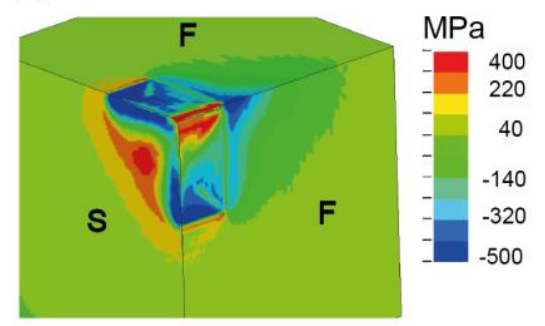

Figure 12 - (a) a corner shot to treat a straight edge
$\sigma_{x x}$ stress distribution
(b) from eigenstrain analysis
(c) stabilised solution from an explicit $\mathrm{FE}$ simulation $(\mathrm{F}=$ Free plane $\mathrm{S}=$ Symmetry plane) 


\section{Explicit finite element analysis of LSP treatment at a rounded (chamfered) edge}

The results in the previous section suggest that only a modest surface compressive stress will be developed in the vicinity of a straight free edge (Figures 8a and 8c). However, a reasonably high sub-surface tension can arise. Furthermore, there are potential surface tensile stresses developed due to the practical difficulty of treating the area adjacent to the edge (Figure 11b). These effects mean that the actual benefit of LSP treatment can be marginal or may even weaken a component. One way to counteract this problem is by introducing a rounded (or chamfered) corner.

In order to determine the effect of LSP treatment on a rounded corner, 16x8x8 mm specimens with edge radii 0.5 and $1.5 \mathrm{~mm}$ were analysed. It was assumed that each corner is peened by the same laser system described above $\left(I=9 \mathrm{GW} / \mathrm{cm}^{2}, p_{\max }=6.7 \mathrm{GPa}, t_{p}=100 \mathrm{~ns}\right)$. An explicit FE simulation was run for each geometry, again employing symmetry about the $y z$ plane. Figures $13 \mathrm{a}$ and $13 \mathrm{~b}$ show the $\sigma_{x x}$ stress distribution in the specimens with $1.5 \mathrm{~mm}$ and 0.5 $\mathrm{mm}$ radii respectively. The results are compared with that in an equivalent specimen with a straight edge (Figure 8a). It can be seen that a desirable residual stress is developed in the specimen with a large radius corner (Figure. 13a) where the surface compressive stress of magnitude $\sim 100-150 \mathrm{MPa}$ is developed in the vicinity of the edge and $\sim 420-450 \mathrm{MPa}$ at locations slightly further away. The corresponding stresses in the small radius corner are 100-120 MPa and 400-420 MPa; and 20-50 MPa and 250-300 MPa at the sharp edge. For each geometry, a peak sub-surface tension of $\sim 150-200 \mathrm{MPa}$ is developed. The depth of the compression zone is $\sim 0.8 \mathrm{~mm}$ in the specimens with rounded corners, whereas at a straight edge a slightly deeper compressive layer $(\sim 1.1 \mathrm{~mm})$ is developed. It may be seen that, a rounded corner helps to achieve a beneficial residual stress field and, as expected the stress field approaches that at a sharp edge as radius is reduced. Whilst an eigenstrain simulation to the problem might be developed, the principal strain directions would vary around the radius, 
making the approach more complex to implement. It has not, therefore, been attempted here. However, a related problem where the radius was introduced after LSP treatment can be simply modelled with the knowledge of the basic eigenstrain distributions used earlier ${ }^{3}$. However, it should be appreciated that this approach assumes that limited additional eigenstrain is introduced when the radius is machined. Depending on the machining method used, this may or may not be a reasonable approximation.

(a)

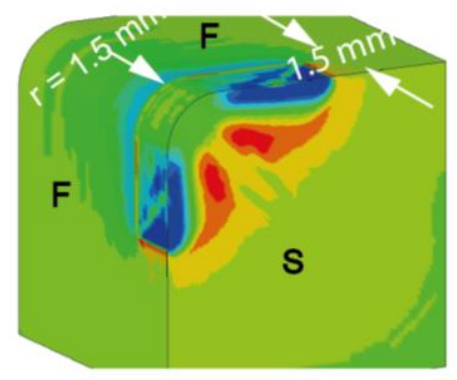

(b)

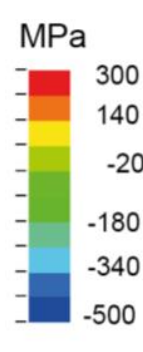

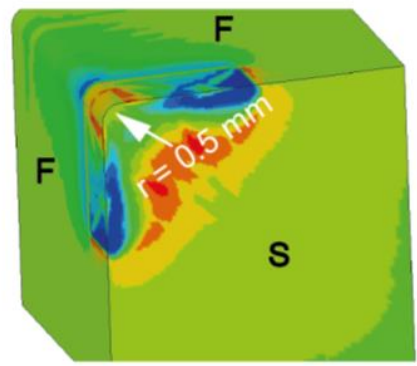

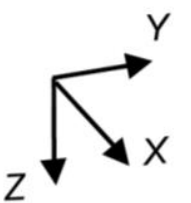

Figure13 $-\sigma_{x x}$ stress distribution at rounded corners with radii $\quad$ (a) $1.5 \mathrm{~mm} ; \quad$ (b) $=0.5 \mathrm{~mm}$ $(\mathrm{F}=$ Free plane $\quad \mathrm{S}=$ Symmetry plane $)$

\section{Analysis of LSP treatment at a compressor blade leading edge}

One interesting application envisaged for the LSP process is to mitigate effects of foreign object damage (FOD) on the leading edges of compressor blades in aircraft engines ${ }^{13}$. FOD is caused by objects (e.g. debris) ingested into engines, and can cause a significant reduction in the fatigue life of the blades ${ }^{14}$. FOD is a significant cause of unscheduled maintenance and repair of engines, and if a compressive residual stress field is introduced, the damage tolerance of the blades can be improved. Hence, simulation of LSP on leading edges (where most FOD is sustained) is important, and we will examine this geometry as our final example.

Explicit finite element analysis of a compressor blade leading edge. As with the previous geometries, we will first carry out an explicit FE simulation, before developing an 
approximate eigenstrain analysis. The particular geometry chosen is a $10 \times 1 \times 20 \mathrm{~mm}$ Ti-6Al4V specimen with a semi-circular edge of diameter $1 \mathrm{~mm}$, peened by an array of LSP shots $\left(I=9 \mathrm{GW} / \mathrm{cm}^{2} ; t_{L}=18 \mathrm{~ns} ; p_{\max }=6.7 \mathrm{GPa}\right.$ and $t_{p}=100 \mathrm{~ns}$ ) (Figure $\left.14 \mathrm{a}\right)$. It is assumed that a $4.5 \times 4.5 \mathrm{~mm}$ wide surface patch on each side around the edge is peened. The selected geometry and LSP arrangement approximate the type of surface treatment normally applied to compressor blades. Again, because of symmetry only a half of the specimen is modelled.

(a)

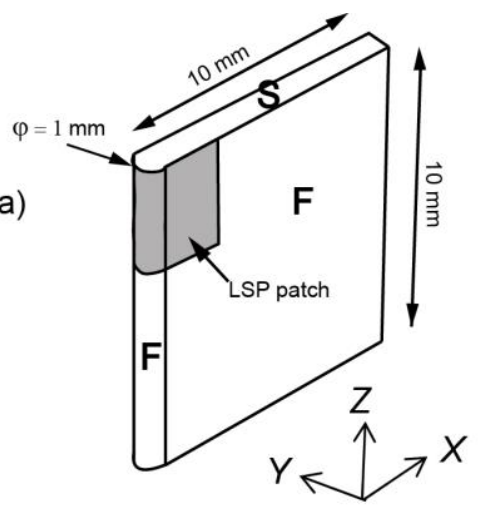

(b)

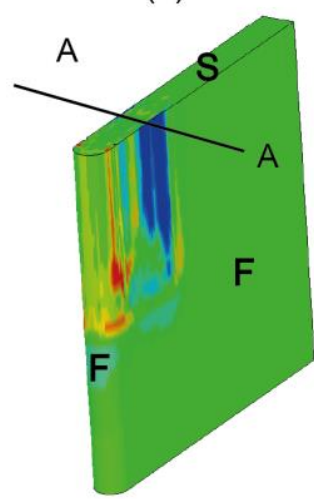

Vertical section through A-A

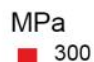

300
140

$-20$

-20
-180

-340
-600

(c)

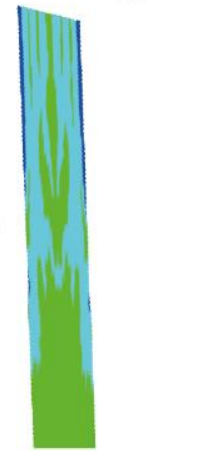

Figure 14 - (a) An simplified geometry for the leading edge of an aircraft engine blade

(b) $\sigma_{z z}$ stress distribution (c) $\sigma_{z z}$ stress variation through a cross section

$$
(\mathrm{F}=\text { Free plane } \quad \mathrm{S}=\text { Symmetry plane })
$$

Figure $14 \mathrm{~b}$ shows the results obtained from the explicit FE analysis for the radial stress $\left(\sigma_{z z}\right)$ distribution, which is usually the most important in blade design. The figure shows that no significant residual stress is developed around the leading edge (the magnitude of the stress here is in the order of $20 \mathrm{MPa}$ (tension) to $50 \mathrm{MPa}$ (compression)). This result highlights that an elastic constraint from the material surrounding the plastic strain caused by LSP is required in order to develop a significant residual stress field. Previous results ${ }^{1-2}$ have shown that an LSP pulse of a typical laser system with energy density of $9 \mathrm{GW} / \mathrm{cm}^{2}$ usually produces a 
plastically deformed zone of $\sim 2.5 \mathrm{~mm}$ deep with a largely constant plastic strain up to $\sim 1.2-$ $1.4 \mathrm{~mm}$ deep in a thick specimen. The leading edge geometry considered in the analysis (Figure 14a) has a thickness of $\sim 1 \mathrm{~mm}$ and the peening was simulated on each side around the edge. Therefore, the assumption of a uniform eigenstrain distribution in the peened area is a reasonable first approximation. Since all the material is subjected to a similar plastic strain no significant residual stress is developed. However, it can be seen from Figure $14 \mathrm{~b}$ that, towards the edge of the LSP patch, a significant surface compressive stress is developed, because of the constraint provided by the neighbouring untreated material. Figure $14 \mathrm{c}$ shows the residual stress distribution through a typical cross section close to the remote edge of the LSP patch (Section A-A in Figure 14b). The results show that the magnitude of the surface compressive stress diminishes gradually until the mid-thickness where no significant stress is present. In conclusion, the results suggest that although an advantageous compressive residual stress will not be developed adjacent to the leading edge, the overall fatigue strength of the whole blade may still be improved due to the compressive stress developed slightly away from the leading edge.

\section{Eigenstrain modelling of a compressor blade leading edge}

The eigenstrain distribution generated under an LSP patch in the vicinity of a leading edge is almost uniform through the entire thickness of the specimen, we can carry out a simple analysis using a constant eigenstrain. Figure 15a shows the $\sigma_{z z}$ stress distribution obtained for the same specimen by incorporating a uniform eigenstrain distribution (i.e. the eigenstrain depth profile shown in Figure $4 \mathrm{c}$ up to depth $1 \mathrm{~mm}$ ) over the required volume within a static FE model. As expected, no significant stress is developed adjacent to the leading edge (Figure 15a). However, as with the explicit analysis, significant surface compressive stresses are developed close to the edges of the LSP patch where the surrounding elastic material provides constraint for the eigenstrain distribution. However, it should be appreciated that the 
eigenstrain model predicts slightly higher peak tension than the explicit FE approach. Furthermore, the high compression region along the edge of the peened area (Figure 15a) itself is not reproduced in the explicit model because of the approximation of the uniform eigenstrain distribution in the in-plane direction (xz plane). The simplified eigenstrain model can be used to study the effect due to various complex LSP treatment arrangements. For instance, Figure $15 \mathrm{~b}$ shows the $\sigma_{z z}$ distribution in the same specimen if the LSP patch is applied only on the front and back surfaces without treating the curved edge fully. As with a sharp $90^{\circ}$ edge (Figure $11 \mathrm{~b}$ ), the results suggest that failure to treat the edge fully causes a significant surface tension in the untreated area. The results also show that due to the elastic misfit exist between the LSP treated zone and the outside unpeened area a significant surface compression forms, similar to that at the other edges of the LSP patch, is developed around leading edge.

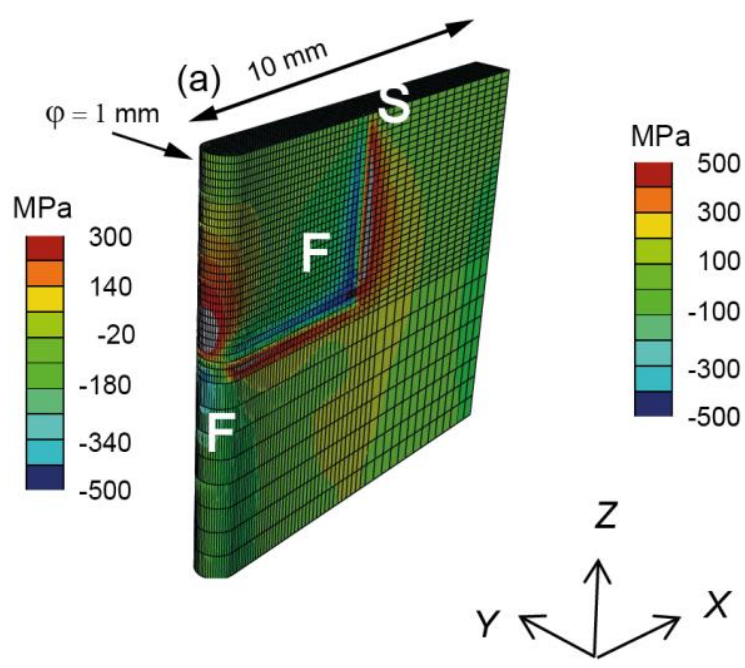

(b)
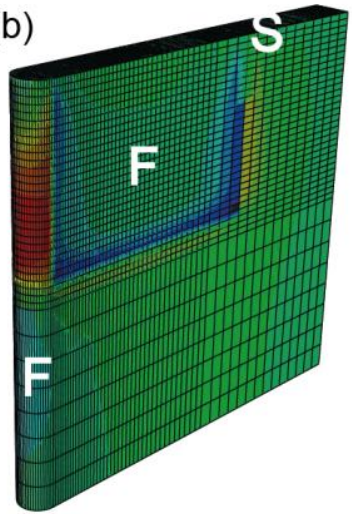

Figure 15 - (a) $\sigma_{z z}$ stress distribution determined from an eigenstrain analysis

(b) surface tension in an untreated curved zone

$$
(\mathrm{F}=\text { Free surface } \quad \mathrm{S}=\text { Symmetry plane })
$$

\section{Conclusions}

The study has used explicit finite element analyses, together with a simple eigenstrain approximation, to model the interaction between the LSP process and geometric features of structural components: sharp and rounded free edges and leading edge geometries. 
The paper shows that the eigenstrain approach can provide good approximations to the residual stress field generated and provides a computationally efficient tool for modelling residual stress generated in practical structural components due to laser shock peening. All that is required is a 'library' of different eigenstrain profiles caused by the application of a range of laser parameters to a single patch on a flat plate.

The results show that the eigenstrain distribution caused by a LSP patch around a free edge can be appropriately modelled by using superposition of that caused by an individual LSP shot in the array. This allows study of the effect of various geometric features in a computationally efficient manner with a reasonable degree of accuracy.

Both explicit and eigenstrain results demonstrate that the interaction between the LSP process and geometric features is important for understanding the subsequent performance of components.

The results show that not all applications of LSP provide an advantageous residual stress field. If a narrow zone adjacent to the free edge remains untreated or experiences an eigenstrain of lower magnitude than elsewhere under the pulse, a very high surface tensile stress can be developed in the material close to an edge. When the edge is treated fully, no constraint is provided by the free edge in response to the plastic strain caused by LSP whereas a narrow zone of elastic material provides constraint against plastic strain when an untreated narrow zone remains adjacent to the free edge. The variation of the constraint in response to the plastic strain results in two different residual stress in these two cases.

The effects of LSP at a rounded edge were also studied and the results show that LSP produces a more advantageous residual stress field than with a sharp edge. Finally, the effect of LSP on fan blade leading edge geometry was also analysed. The results for this geometry suggest that, although a significant compressive residual stress will not be developed adjacent to a leading edge, the overall fatigue strength of a component may still be improved by LSP due to the compressive stress field developed further away from the leading edge. Residual 
compression can persist through the thickness of relatively thin sections such as this, with the balancing tension carried further away from the leading edge in the unpeened region.

Only a limited range of component geometries are considered in the current study since the primary objective of the study was to analyse the effect of LSP on leading edge geometries in fan blades of aircraft engines. Analyses of the effect of LSP on other geometries such as spheres are not considered in this paper. In any case, the analysis of LSP on a sphere will be the same as that of the analysis of a plane sample, if the patch is small compared to the radius. If it is large, then one would need to be concerned about how the sphere is supported, so the analysis is not straightforward as other geometries discussed in the present paper.

\section{Acknowledgements}

The financial support of the Engineering and Physical Sciences Research Council (EPSRC) and the UK Ministry of Defence (Grant Number EP/F026226/1) is gratefully acknowledged.

\section{References}

[1] Achintha M and Nowell D. 2011. Eigenstrain modelling of residual stresses generated by laser shock peening. J Mater Process 2011; 211: 1091-1101.

[2] Achintha M, Nowell D, Shapiro K, et al. 2013. Eigenstrain modelling of residual stress generated by arrays of Laser Shock Peeing shots and determination of the complete stress field using limited strain measurements. Surf Coati Technol 2013; 216: 68-77.

[3] Achintha M, Nowell D, Fufari D, et al. Fatigue behaviour of geometric features subjected to laser shock peening: experiments and modelling. Int J Fatigue 2014; 62: 171-179.

[4] Ding $\mathrm{K}$ and Ye L. Laser shock peening: Performance and process simulation., Cambridge: Woodhead, 2006.

[5] Fabbro R, FournierJ, Ballard P, et al. Physical study of laser-produced plasma in confined geometry. J Appl Phys 1990; 68: 775-784. 
[6] Kim JY, Shim IO, Kim HK, et al. Dynamic deformation and high velocity impact behaviours of Ti-6Al-4V Alloys. Mater sci Forum 2007; 539-543:2269-2274.

[7] McGeachie I. 2010. Private Communication, Metal Improvement Company, Earby, UK.

[8] Berthe L, Fabbro R, Peyre P, et al. 1997. Shock wave from a water-confined lasergenerated plasma. J. Appl. Phys 1997: 82 (6): 2826-2832.

[9] LS-DYNA (V971), 2007. Livermore Software Technology Corporation (LSTC)

[10] Brockman RA, Braisted WR, Olson SE, et al. Prediction and characterization of residual stresses from laser shock peening. Int J Fatigue 2012; 36: 96-108.

[11] Dorman M, Toparli MB, Smyth N, et al. Effect of laser shock peening on residual stress and fatigue life of clad 2024 aluminium sheet containing scribe defects. Mater Sci Eng: A 2012; 548: 142-151.

[12] Abaqus 6.10, 2009. SIMULIA

[13] Spanrad S and Tong J.Characterisation of foreign object damage (FOD) and early fatigue crack growth in laser shock peened Ti-6Al-4V aerofoil specimens. Mater Sci Eng A 2011; 528: $2128-2136$.

[14] Mall S, Hamrick JL, Nicholas T. 2001. High cycle fatigue behaviour of Ti-6Al-4V with simulated foreign object damage. Mechanics of Materials 2001; 33: 679-692. 


\section{List of Figure captions}

Figure 1 (a) - An edge is treated from both the directions to achieve a desired residual stress

$$
(\mathrm{F}=\text { Free plane; } \mathrm{S}=\text { Symmetry plane })
$$

(b) - Assumed variation of pressure with time, caused by application of a laser pulse

Figure 2 - Stabilised residual stress distribution determined from a completely explicit FE

$$
\text { simulation } \quad\left(\begin{array}{lll}
\text { (a) } \sigma_{x x} & \text { (b) } \sigma_{\mathrm{yy}} & (\mathrm{F}=\text { Free plane, } \mathrm{S}=\text { Symmetry plane })
\end{array}\right.
$$

Figure 3 - A single LSP shot pulsed adjacent to a straight free edge

$$
(\mathrm{F}=\text { Free plane } \mathrm{S}=\text { Symmetry plane })
$$

Figure 4 - Eigenstrain distribution under an (a) edge pulse (b) central pulse

(c) eigenstrain depth profile at the centre point of an edge pulse and a central pulse

$$
(\mathrm{F}=\text { Free plane } \quad \mathrm{S}=\text { Symmetry plane })
$$

Figure 5 - A single LSP pulse with an offset from the free edge

$$
(\mathrm{F}=\text { Free plane } \quad \mathrm{S}=\text { Symmetry plane })
$$

Figure 6 - Eigenstrain distribution under an LSP shot with an offset of (a) $0.15 \mathrm{~mm}$

(b) $0.3 \mathrm{~mm}$ from the free edge $\quad$ (c) eigenstrain depth profile at the centre of an edge shot and that at a central shot $(\mathrm{F}=$ Free plane $\mathrm{S}=$ Symmetry plane $)$

Figure 7 - (a) $\varepsilon_{x x}^{p}$ (b) $\varepsilon_{y y}^{p}$ (c) $\varepsilon_{z z}^{p}$ principal components of the eigenstrain (d) eigenstrain depth profile at the centre of the pulse after the $1^{\text {st }}$ pulse and after the $2^{\text {nd }}$ pulse

$$
(\mathrm{F}=\text { Free plane } \quad \mathrm{S}=\text { Symmetry plane })
$$

Figure 8 - Residual stress distribution: (a) $\sigma_{x x}$ stress at the edge (from eigenstrain model)

(b) comparison between the $\sigma_{x x}$ stress component predicted from the eigenstrain method and from explicit analysis (c) $\sigma_{y y}$ stress at the edge (eigenstrain model) $(\mathbf{d}) \sigma_{x x}\left(=\sigma_{y y}\right)$ under a central LSP patch (eigenstrain model) $\quad(\mathrm{F}=$ Free plane $\quad \mathrm{S}=$ Symmetry plane $)$

Figure 9 - Explicit FE results for the eigenstrain distribution (a) if the two shots were applied simultaneously (b) if the second shot was applied after the residual stress field due to the first pulse had stabilised (c) Residual stress depth profile at a point $0.5 \mathrm{~mm}$ from the free edge $(\mathrm{F}=$ Free plane $\mathrm{S}=$ Symmetric plane $)$ 
Figure 10 - (a) Treatment of a straight edge using an LSP strip

$\sigma_{x x}$ stress distribution under the (b) short $(\mathrm{L}=9 \mathrm{~mm})$ strip (c) long $(\mathrm{L}=27 \mathrm{~mm})$

strip ( $\mathrm{F}=$ Free plane $\mathrm{S}=$ Symmetry plane)

Figure 11 - (a) A narrow untreated strip adjacent to the free edge.
Residual stress distribution
(b) $\sigma_{x x}$
(c) $\sigma_{y y}$

$$
(\mathrm{F}=\text { Free plane } \quad \mathrm{S}=\text { symmetric plane })
$$

Figure 12 - (a) a corner shot to treat a straight edge

$$
\begin{aligned}
& \sigma_{x x} \text { stress distribution (b) from eigenstrain analysis (c) stabilised solution } \\
& \text { from an explicit } \mathrm{FE} \text { simulation }(\mathrm{F}=\text { Free plane } \quad \mathrm{S}=\text { Symmetry plane })
\end{aligned}
$$

Figure13 $-\sigma_{x x}$ stress distribution at rounded corners with radii $\quad$ (a) $1.5 \mathrm{~mm} ; \quad$ (b) $=0.5 \mathrm{~mm}$

$$
(\mathrm{F}=\text { Free plane } \quad \mathrm{S}=\text { Symmetry plane })
$$

Figure 14 - (a) An simplified geometry for the leading edge of an aircraft engine blade

(b) $\sigma_{z z}$ stress distribution (c) $\sigma_{z z}$ stress variation through a cross section

$$
(\mathrm{F}=\text { Free plane } \quad \mathrm{S}=\text { Symmetry plane })
$$

Figure 15 - (a) $\sigma_{z z}$ stress distribution determined from an eigenstrain analysis

(b) surface tension in an untreated curved zone

$$
(\mathrm{F}=\text { Free surface } \quad \mathrm{S}=\text { Symmetry plane })
$$

